'Laboratorio de Genética y Enfermedades Metabólicas. Instituto de Nutrición y Tecnología de los Alimentos, Universidad de Chile.

${ }^{2}$ Servicio de Pediatría, Hospital Base de Puerto Montt.

'Laboratorio Clínico, Hospital Base de Puerto Montt. ${ }^{4}$ Servicio de GinecoObstetricia, Hospital Base de Puerto Montt.

${ }^{5}$ Servicio de Neonatología, Hospital Base de Puerto Montt.

${ }^{6}$ Servicio de Pediatría, Hospital Regional de Valdivia.

Estudio Colaborativo Latinoamericano de Malformaciones Congénitas (ECLAMC).

${ }^{a}$ Magíster en Ciencias Biológicas c/m en Genética.

Universidad de Chile. ${ }^{\text {b} T e c n o ́ l o g o ~ M e ́ d i c o . ~}$ Universidad de Chile 'Doctor en Ciencias c/m en Biología Celular, Molecular y Neurociencias. Universidad de Chile.

Recibido 9 el junio de 2010, aceptado el 17 de enero de 2011

Correspondencia a: M. Angélica Alliende R. Laboratorio de Genética y Enfermedades Metabólicas, INTA, Universidad de Chile. Av. El Líbano 5524, Macul. Casilla 138 Santiago 11.

Santiago de Chile. Fax: 56(2) 978-1489. E-mail: malliend@inta. uchile.cl

\section{Caracterización citogenético-molecular de enfermedades genéticas en el Hospital Base de Puerto Montt}

\author{
M. ANGÉLICA ALLIENDE ${ }^{1, \mathrm{a}}$, BIANCA CUROTTO $^{1, \mathrm{~b}}$, PATRICIO GUERRA $^{2}$, \\ LORENA SANTA MARÍA ${ }^{1, \mathrm{c}}$, REINERÍA HERMOSILLA ${ }^{3}$, \\ DORIS ORPHANÓPOULOS ${ }^{4}$, JORGE VILLANUEVA ${ }^{2}$, \\ ELIZABETH WETTIG ${ }^{5,7}$, XIMENA BARRAZA $^{6}$
}

\section{Cytogenetic and molecular profile of genetic diseases in Puerto Montt main hospital}

\begin{abstract}
Background: Chromosome aberrations (CA) are the main etiology of multiple congenital malformations, recurrent abortions and intellectual disability (ID) specifically of moderate and severe degree. They account for 0.3 to $1 \%$ of newborns (NB) and 6 of 10,000 NB have chromosome imbalances with submicroscopic deletions or duplications smaller than $10 \mathrm{MB}$ that are overlooked by conventional cytogenetic studies. Aim: To report the results of cytogenetic and molecular studies performed in patients with a congenital malformation disease or ID with or without dysmorphic features, attended in a regional hospital. Patients and Methods: One hundred and eighty patients, 27 with a clinical diagnosis of Down syndrome, derived for the suspicion of a genetic disease, were studied. A karyogram was performed in all of them and in 30 cases additional molecular studies, such as fluorescence in situ hybridization (FISH) or polymerase chain reaction (PCR) were carried out. Results: Among the 153 patients without Down syndrome, 20 (13\%) had a genetic abnormality responsible for the altered phenotype. Sixteen had a chromosome aberration (structural and numerical aberrations in 75 and 25\% respectively) and four had genetic molecular alterations. Additional studies were performed to confirm or better characterize the chromosome aberration in 13 of the 30 patients in whom these were requested. Conclusions: Chromosome and specific genetic molecular studies in selected cases help to characterize patients with genetic diseases. The collaboration between academic and health care facilities is crucial.
\end{abstract}

(Rev Med Chile 2011; 139: 298-305).

Key words: Chromosome Abnormality disorders; Cytogenetic analysis; Molecular genetics.

$\mathrm{E}$ xisten múltiples factores que pueden influir negativamente en la sobrevida del embrión, sin embargo, las aberraciones cromosómicas (AC) son una de las más significativas, ya que alteran la expresión de muchos genes importantes en etapas tempranas del desarrollo embrionario. Las AC de tipo numérico o aneuploidías y estructurales son las principales causas de mal- formaciones congénitas, presentándose en $60 \%$ de los abortos espontáneos, $12 \%$ de las parejas con infertilidad y en $6 \%$ de los nacidos vivos con malformaciones. No es sorprendente entonces que todas las pérdidas de cromosomas autosómicos y la mayoría de las trisomías produzcan pérdidas fetales tempranas ${ }^{1,2}$.

La prevalencia estimada de $\mathrm{AC}$ al nacimiento es 
de 0,3 al 1\% y, en nuestro país, entre recién nacidos (RN) malformados es de 4,3\%, con una distribución similar (cercana a 50\%) entre aneuploidías y anomalías estructurales ${ }^{3,4}$. En algunos casos, éstas son sugerentes de un síndrome conocido y en otros, debido a la variabilidad del fenotipo y la severidad de sus malformaciones, pueden ser tan diversas como las posibilidades de reordenamientos cromosómicos existentes, incluso podrían estar asociadas a enfermedades complejas y a cáncer ${ }^{5,6}$.

Se ha estimado que 0,5 a 1 en 10.000 de los recién nacidos tienen una deleción cromosómica constitucional visible al microscopio de luz, incluidos los síndromes cromosómicos clásicos, como las trisomías 21, 18 y 13; sin embargo, cerca de 6 en 10.000 RN tienen un desbalance cromosómico que corresponde a deleciones o duplicaciones de un tamaño menor a 10 megabases $(\mathrm{Mb})$ y que sólo es posible de detectar por métodos moleculares ${ }^{7}$. Por esto, desde 1996, los estudios genéticos con fines diagnósticos incluyen técnicas de genética molecular, dotadas de una mayor sensibilidad, en particular hibridación in situ con fluorescencia (FISH), reacción de polimerasa en cadena (PCR), secuenciación de genes, hibridación comparativa del genoma (CGH) y microarrays ${ }^{7-9}$. Estas metodologías han permitido detectar un número creciente de anomalías cromosómicas crípticas, constituyéndose así el "cariotipo molecular" como una estrategia complementaria que aporta mayor resolución al análisis del cariotipo convencional ${ }^{10-12}$.

La prevalencia del retraso mental o déficit intelectual (DI) en la población general es 2 a $3 \%$, con variaciones entre 1 y $10 \%$ según el tipo de población estudiada ${ }^{13-15}$. Considerando que las aberraciones cromosómicas son la principal causa etiológica de DI, especialmente en los grados moderado a severo, es que el DI es otra de las principales indicaciones para análisis cromosómico y búsqueda de mutaciones relativamente frecuentes, como las responsables de los síndromes X frágil, Prader Willi y Angelman, entre otros. Identificar la etiología de estas alteraciones permite el inicio precoz del manejo clínico adecuado, la estimulación sensorial y motora dirigida; además conocer el pronóstico y riesgo de recurrencia de la afección, así como también poder entregar asesoría genética reproductiva oportuna a los padres y otros familiares a riesgo ${ }^{16-18}$.

Lo anteriormente expuesto y la evidencia de una tasa de mortalidad 11 veces mayor en pa- cientes portadores de una deleción cromosómica constitucional que en la población general, así como que las alteraciones genéticas ocupan en este momento el segundo lugar como causa de mortalidad infantil, después de la prematurez extrema, avalan la necesidad de estudiar un número creciente de pacientes para el diagnóstico de afecciones genético-cromosómicas ${ }^{6,19,20}$. Esta realidad ha motivado a los sistemas de salud públicos y privados a implementar nuevos laboratorios de citogenética con metodologías moleculares que permitan encontrar la causa de un número también creciente de afecciones genéticas, evitando de esta manera la realización de otros estudios más invasivos y costosos ${ }^{21}$.

Es difícil comparar el rendimiento de los laboratorios de citogenética, medido en términos de estudios cromosómicos alterados con respecto del total de exámenes. En las diferentes publicaciones existen variaciones importantes según los diagnósticos de referencia, criterios utilizados al momento de solicitar el examen, tipo de pacientes estudiados y la metodología utilizada. Además, la experiencia demuestra que al disminuir la estrictez en los criterios de selección de los pacientes, aumenta el diagnóstico de las anomalías cromosómicas estructurales como deleciones, translocaciones, isodicéntricos y otros ${ }^{7,8}$.

Esta variabilidad también se observa al analizar la experiencia publicada en Chile. Al respecto, dos estudios realizados en las Regiones II y Metropolitana respectivamente, considerando muestras similares, compuestas por recién nacidos con malformaciones congénitas, lactantes, preescolares y escolares con síndromes dismórficos, ambigüedad genital y otras genopatías, excluyendo el síndrome de Down (SD), mostraron alteraciones cromosómicas en $9,5 \%{ }^{22}$ y en $19,3 \%{ }^{23}$ respectivamente. Otro estudio, realizado en la Región Metropolitana y menos restrictivo en la selección de los pacientes, consideró pacientes adultos con pérdida reproductiva, disgenesia gonadal o menopausia precoz y otros diagnósticos, mostró 8,4\% de casos altera$\operatorname{dos}^{24}$. En todos ellos se utilizó sólo la citogenética convencional en la obtención de los resultados; la selección de los pacientes fue establecida por un genetista y la estrictez en la indicación de examen se relacionó con el costo y la disponibilidad de un laboratorio especializado.

La población atendida en el Hospital Base de Puerto Montt (HBPM), en la X Región, incluidas 
las provincias de Llanquihue, Chiloé y Palena, se ha caracterizado consistentemente por presentar frecuencias inusualmente altas de pacientes con DI con y sin dismorfias, en los cuales se sospecha una afección genético-cromosómica (observaciones no publicadas); sin embargo, en la mayoría de los casos no se realizan los estudios que permiten establecer el origen de la afección, por la imposibilidad de contar en la zona con laboratorios con la tecnología y experiencia adecuada para este tipo de diagnóstico.

Este trabajo pretende mostrar la importancia de desarrollar estudios colaborativos entre laboratorios de genética que permitan ampliar y optimizar los recursos destinados al diagnóstico de pacientes atendidos por el sistema público de salud. Esto es especialmente relevante cuando la sospecha clínica, de ciertas enfermedades genéticas, indica la realización de estudios citogenéticos y genético-moleculares complementarios, para su confirmación diagnóstica.

\section{Pacientes y Métodos}

\section{Pacientes}

Se estudiaron 180 pacientes derivados al Laboratorio Clínico del HBPM: 92 hombres y 88 mujeres, distribuidos en los siguientes grupos etarios: 58 recién nacidos y lactantes de 0 a 1 año; 55 preescolares y escolares de 1 a 8 años; 39 adolescentes de 8 a 14 años y 28 mayores de 14 años y adultos. Del total de pacientes, 27 tenían diagnóstico clínico de síndrome de Down; los 153 restantes fueron derivados por distintos especialistas, no genetistas, con diagnósticos clínicos de: genopatía o múltiples malformaciones congénitas $(\mathrm{N}=39)$; retraso mental y retraso del desarrollo psicomotor con y sin dismorfias $(\mathrm{N}=36)$; talla baja en estudio, amenorrea primaria o ambos $(\mathrm{N}=34)$; abortos a repetición e historia familiar de aberración cromosómica $(\mathrm{N}=10)$; síndrome $\mathrm{X}$ frágil $(\mathrm{N}=8)$; espectro DiGeorge $($ del $22 \mathrm{q})(\mathrm{N}$ $=8$ ) hipotonía neonatal $(\mathrm{N}=6)$, síndromes de Prader Willi y Angelman (SPW/SA) $(\mathrm{N}=6)$ y otros $(\mathrm{N}=6)$.

\section{Metodología}

Estudios citogenéticos: En los 180 casos se determinó el cariotipo a partir del análisis cromosómico con bandeo GTG, en cultivo de linfocitos de sangre periférica. La obtención de cromosomas se realizó en el Laboratorio Clínico del HBPM; el recuento, microfotografías y análisis del cariotipo se realizaron en el Laboratorio de Citogenética del INTA, utilizando metodología previamente publicada $^{18}$.

Estudios moleculares complementarios: Del total de casos estudiados, en 30 de ellos el diagnóstico clínico presuntivo sugirió los siguientes análisis moleculares complementarios: estudio de síndromes de microdeleción por FISH de secuencia única, en 16 pacientes; por sospecha clínica de síndrome de Prader Willi (SPW) y Angelman (SA) se realizó test de metilación en 6 casos; por sospecha clínica de síndrome X frágil (SXF) se realizó PCR específico en 4 pacientes; 2 casos de DI inespecífico se estudiaron con FISH de regiones subteloméricas; se realizó análisis del gen SRY por PCR en 1 paciente con fenotipo Turner; se realizó PCR múltiple con STRs de la región 15q11-13, para determinar SPW por disomía uniparental (DUP) en 1 caso con test de metilación alterado y FISH normal. En todos ellos se utilizó metodología establecida en el laboratorio del INTA. Brevemente, los estudios de FISH de secuencia única para cromosomas 7,15, 22, X e Y, así como de regiones subteloméricas se realizaron en metafases obtenidas de cultivos de linfocitos, utilizando sondas fluorescentes específicas (Vysis) y visualizadas al microscopio de fluorescencia (Olympus). Para los tests de metilación y PCRs se aisló ADN del paciente utilizando un kit de extracción (Wizard ${ }^{\circledR}$ Genomic DNA Purification, Promega) y se procedió a realizar los estudios respectivos según protocolos establecidos y adaptados en el laboratorio del INTA ${ }^{25}$; para el test de metilación se utilizó el protocolo establecido por Kosaki y cols, $1997^{26}$ y para el diagnóstico del SXF los protocolos de Saluto y $\operatorname{cols}^{27}$ y Tassone y $\operatorname{cols}^{28}$.

\section{Resultados}

\section{Citogenéticos}

De los 180 pacientes estudiados, 135 resultaron normales y en 45 (25\%) de ellos se diagnosticó una enfermedad genético-cromosómica. En la Tabla 1 se detalla el tipo de aberración cromosómica numérica o estructural y las alteraciones moleculares detectadas.

Del total de pacientes, 27 (15\%) tenían diagnóstico clínico de síndrome de Down. En 24 (88\%) 
Tabla 1. Tipos de alteraciones cromosómicas y moleculares detectadas en los $\mathbf{1 8 0}$ pacientes, según rango de edad

\begin{tabular}{|c|c|c|c|c|}
\hline \multirow[t]{2}{*}{$\begin{array}{l}\text { Edad } \\
\text { (años) }\end{array}$} & \multirow[t]{2}{*}{$\mathbf{n}$} & \multicolumn{3}{|c|}{$\begin{array}{l}\text { Resultados alterados } \\
\qquad n=45\end{array}$} \\
\hline & & $\begin{array}{l}\text { Alteraciones } \\
\text { numéricas }\end{array}$ & Alteraciones estructurales & $\begin{array}{l}\text { Alteraciones } \\
\text { moleculares }\end{array}$ \\
\hline 0 a 1 & 58 & $\begin{array}{l}47, X X,+21(n=13) \\
47, X Y,+21(n=9)\end{array}$ & $\begin{array}{l}46, X X, \text { add }(14)(\text { pter } \rightarrow q 32:: ?) d n \\
46, X Y, \text { add }(16)(p t e r \rightarrow q 24:: ?)^{*} \\
46, X Y,+21, \operatorname{der}(21 ; 21)(q 10 ; q 10) d n \\
46, X Y, \operatorname{der}(2) t(2 ; 15)(q 37 ; q 22) \text { pat }\end{array}$ & \\
\hline$>1$ a 8 & 55 & $\begin{array}{l}45, X \\
47, X Y,+21(n=2) \\
47, X X Y \\
47, X Y Y\end{array}$ & $\begin{array}{l}46, X Y, t(1 ; 12)(p 31 ; q 12) d n \\
47, X Y, \operatorname{dup}(18)(q 21.2)+\operatorname{mar}[11] / \\
46, X Y, \operatorname{dup}(18)(q 21.2)[10]^{a}\end{array}$ & SPW ( DUPmat) $)^{a, b, c}$ \\
\hline$>8$ a 14 & 39 & $\begin{array}{l}\operatorname{mos} \\
47, X Y,+21[2] / 46, X Y[48]\end{array}$ & $\begin{array}{l}\operatorname{mos} 45, X[15] / 46, X, i(Y)(q 10)[43]^{a, d, e} \\
\operatorname{mos} 45, X[42] / 46, X, i(X)(q 10)[8]^{a} \\
46, X X, \operatorname{dup}(1)(q 2 ?) d n\end{array}$ & $\mathrm{PKU}+\mathrm{MPS}$ \\
\hline$>14$ & 28 & $45, x$ & $\begin{array}{l}\operatorname{mos} 46, X, \operatorname{dic}(X),(p t e r \rightarrow q 25:: \text { cen } \rightarrow \text { pter }) \\
{[43] / 45, X[7] \text { a,e }} \\
\operatorname{mos} 45, X[14] / 46, X, i(X)(q 10)[36]^{a} \\
46, X Y, t(2 ; 15)(q 37 ; q 22)^{*}\end{array}$ & $\begin{array}{l}46, X Y \text { (mujer) S.I.A } \\
\text { S.A (del(15)(q11-13)) })^{a, b}\end{array}$ \\
\hline Total & 180 & 29 & 12 & 4 \\
\hline
\end{tabular}

*Falta el estudio de los padres; dn = de novo (nueva mutación); DUPmat = Disomía uniparental del cromosoma 15 de origen materno; PKU+MPS = Paciente con ambas mutaciones: fenilquetonuria y mucopolisacaridosis; S.I.A= Síndrome de insensibilidad androgénica; S.A = Síndrome de Angelman. Estudios Moleculares: ${ }^{\mathrm{a} F I S H ;}$ ' ${ }^{\mathrm{P}} \mathrm{PCR}$ Test de Metilación; 'PCR Múltiple con STRs del cromosoma 15; dPCR gen SRY; eFISH Subtelomérico.

de ellos se diagnosticó una trisomía 21 libre, en un caso había una trisomía 21 por translocación $(21 ; 21)$ de novo, en otro caso la trisomía 21 estaba en mosaico y sólo en 1 caso se descartó el síndrome de Down, por presentar un cariotipo normal.

De los 153 pacientes restantes, no Down, en 20 casos (13\%) se identificó una alteración: 12 (60\%) aberraciones cromosómicas estructurales, $4(20 \%)$ aneuploidías y $4(20 \%)$ alteraciones genético-moleculares.

Entre las 16 AC diagnosticadas, 12 (75\%) correspondían a alteraciones estructurales, incluyendo translocaciones recíprocas, cromosomas derivativos, isocromosomas, cromosoma dicéntrico y una duplicación (Figura 1). En los 4 casos con translocaciones recíprocas se estudiaron ambos padres, en 3 de ellos la alteración era de novo (dn) y en 1 caso ésta era heredada del padre. Las 4 (25\%) alteraciones numéricas correspondieron a aneuploidías de los cromosomas sexuales (Tabla 1).

\section{Moleculares}

De un total de 30 estudios de genética molecular realizados, 17 fueron normales y 13 (43\%) permitieron confirmar o a caracterizar mejor la aberración cromosómica.

En 6 pacientes con características clínicas de síndrome de Turner, el análisis por FISH permitió en 3 casos caracterizar las alteraciones estructurales del cromosoma X (Figura 2); en 2 casos precisar la constitución del mosaico y en un caso fue posible caracterizar un derivativo del cromosoma $\mathrm{Y}$, como un isocromosoma del brazo largo del Y (Tabla 1, Figura 3).

El test de metilación confirmó en 2 casos los diagnósticos clínicos de síndromes de Angelman y Prader Willi. En el caso con SA el FISH determinó una deleción en 15q11-13 y en el caso con SPW el FISH resultó normal; en este caso el PCR múltiple con STRs de la región 15q11-13 determinó una disomía uniparental 15 materna. 


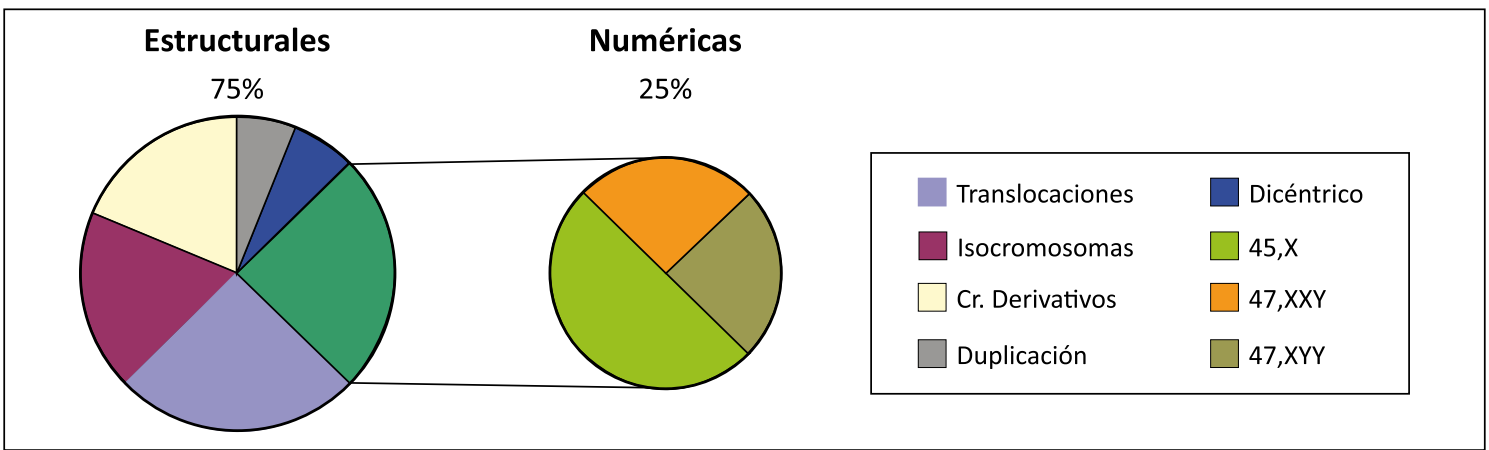

Figura 1. Distribución de alteraciones cromosómicas estructurales y numéricas en 16 casos, excluyendo el Síndrome de Down.

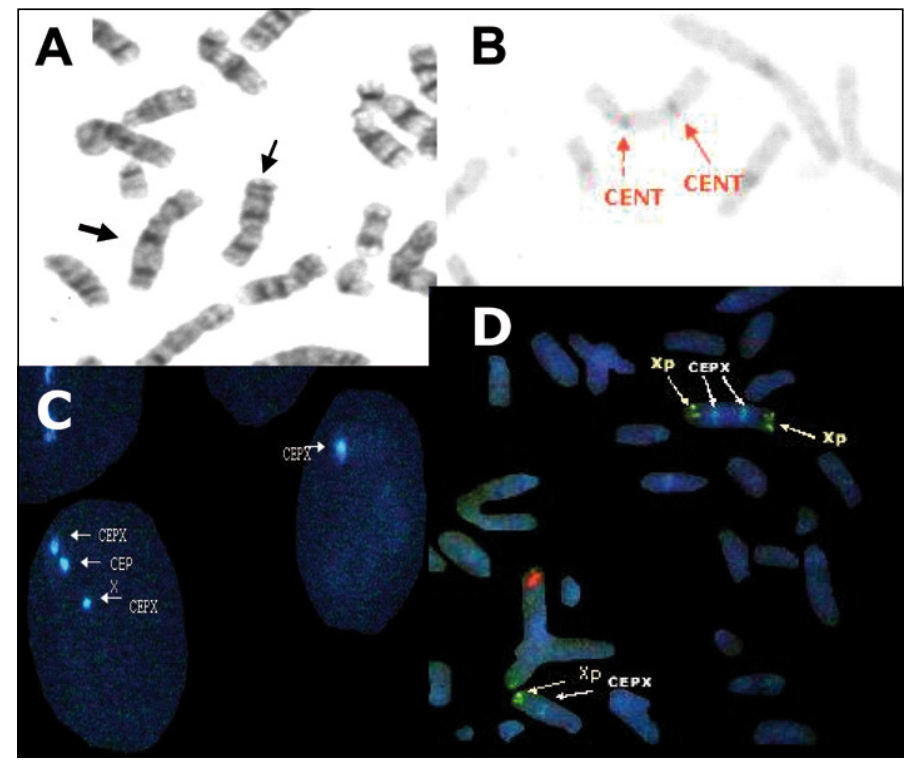

Figura 2. Análisis citogenético y FISH del caso mos 45,X[7]/46,X,der(X)[43]. A: Cariotipo parcial con bandeo GTG. B: Bandeo CBG; las flechas muestran un $\mathrm{X}$ normal y un $\operatorname{der}(\mathrm{X})$. C: FISH de una metafase parcial con sonda centromérica (CEPX): se observan células con una y con 3 señales centroméricas para X. D: FISH con sonda subtelomérica. El cromosoma $X$ normal muestra 1 señal amarilla en la región $p$ subtelomérica y 1 señal verde centromérica ( $X$ cen). El cromosoma $\operatorname{der}(X)$ muestras 2 señales amarillas en la región p subtelomérica y 2 señales centroméricas verde, permitiendo reconocer en el $\operatorname{der}(X) 2$ centrómeros, dos brazos $p$ y deleción parcial del brazo largo desde Xq25 a Xq terminal.

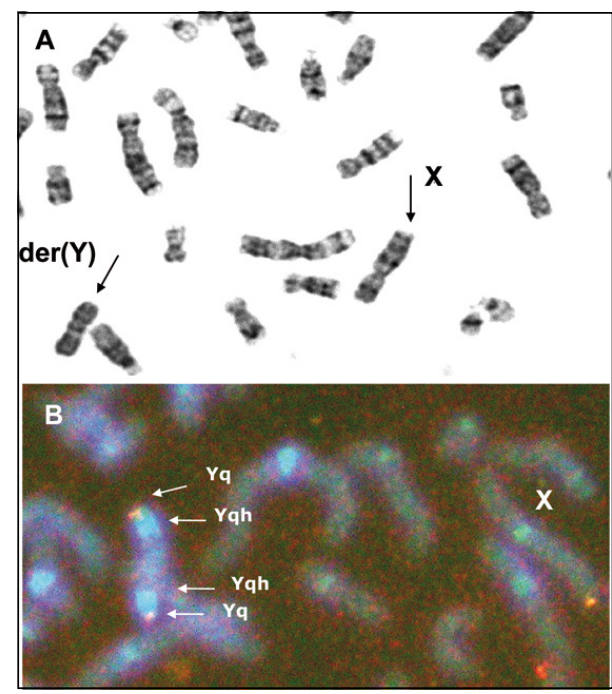

Figura 3. Análisis citogenético y FISH del caso mos45,X[15]/46,X, der(Y) (q10)[43]. A: Cariotipo parcial con bandeo GTG: las flecha muestran un $X$ normal y el derivativo Y. B: FISH de una metafase parcial con sonda de la región subtelomérica Yq; el $\operatorname{der}(Y)$ muestra 2 señales amarillas para la región Yq terminal (ambos extremos) y 2 bloques de heterocromatina DAPI positivo (interior), permitiendo caracterizar el $\operatorname{der}(\mathrm{Y})$ como isocromosoma Yq. 


\section{Discusión}

El análisis de los resultados de este estudio demuestra que de un total de 180 pacientes, en 45 (25\%) de ellos se determinó una afección cromosómica o de genética molecular; en los casos en que los estudios resultaron normales y se descartaron las AC estudiadas, fue posible enfocar la búsqueda a otros factores etiológicos.

De acuerdo a lo esperado, entre los casos con clínica de síndrome de Down, se detectó mayoritariamente (88\%) una trisomía 21 libre; las otras alteraciones como las trisomías en mosaico y por translocación, apoyan la importancia de estudiar todo paciente con fenotipo de SD, ya que además de confirmar la alteración, detecta translocaciones que pueden ser heredadas. Esta situación hace necesario estudiar a los padres para entregar una asesoría genética adecuada.

A pesar que $90 \%$ de los pacientes aquí estudiados no fueron referidos por un genetista, es importante destacar el alto porcentaje de casos (13\%) con alteraciones genéticas, no síndrome de Down. Al comparar estos resultados con otras dos experiencias realizadas en Chile, se observan importantes diferencias: $13 \%$ vs $8,4 \%$ y $9,5 \%$ respectivamente ${ }^{22,24}$. Así también llama la atención una distribución distinta a lo esperado de alteraciones estructurales y numéricas ( $75 \%$ vs $25 \%$ ), (Figura 1); siendo las aneuploidías de los cromosomas $\mathrm{X}$ las únicas detectadas entre las numéricas. En estudios previos se ha establecido que la proporción de anomalías estructurales y numéricas es cercana a $50 \%$ y que las trisomías de los autosomas, incluida la trisomía 21 son las más frecuentes ${ }^{4,7,23}$.

Estas diferencias podrían deberse a una selección estricta de los pacientes aquí estudiados, ya que en su mayoría cumplían con las características clínicas más sugerentes de los síndromes que configuran las indicaciones del estudio cromosómico ${ }^{29,30}$. Este hecho se traduce en un mayor rendimiento de estos estudios, sin embargo, conlleva el riesgo de subdiagnosticar anomalías cromosómicas y genéticas en pacientes que presenten características menos evidentes; los cuales podrían ser pesquisados si se cuenta con mayor facilidad de acceso a estos exámenes, al momento de indicar el estudio a los pacientes.

Existen estudios previos que relacionan el aislamiento, ruralidad y consanguinidad de poblaciones chilenas como la de Chiloé con la aparición de afecciones raras monogénicas del tipo autosómicas recesivas ${ }^{31,32}$. Sin embargo, en este estudio se observa una distribución inusual de aberraciones numéricas y estructurales, especialmente ocurridas "de novo" (Figura 1); esto permitiría postular que en esta zona geográfica existirían factores ambientales o genéticos que podrían estar influyendo en la etiología y un riesgo mayor de afecciones genético-cromosómicas, como ha sido descrito para otras poblaciones y que deberían ser estudiados ${ }^{33,34}$.

En este estudio se observaron diferencias con respecto a las alteraciones visibles sólo a nivel molecular $(\mathrm{N}=4)$, las cuales no fueron pesquisadas en los estudios anteriormente realizados en Chile; debido a que no se disponía entonces del uso habitual de metodologías moleculares complementarias ${ }^{22-24}$. Este es el primer estudio en Chile que muestra alteraciones detectadas con estudios moleculares, en un número importante de casos, todos ellos atendidos en el sistema público de salud de la X Región; además avala la importancia de hacer análisis más resolutivos en pacientes con fenotipos sugerentes de afecciones genéticas, tal como había sido descrito recientemente ${ }^{10,11,18}$.

Los resultados de este estudio avalan la importancia de este tipo de trabajos colaborativos como el que se ha establecido entre el INTA de la Universidad de Chile y el Hospital Base de Puerto Montt. Esto ha permitido facilitar el acceso al diagnóstico etiológico de enfermedades genéticas complejas, evitándose el traslado del paciente a otro centro asistencial, muchas veces del sistema privado, para su estudio. Este estudio también ha significado un enorme beneficio tanto para los pacientes de la región como para el equipo médico del HBPM, por la posibilidad de iniciar tempranamente terapias de estimulación, evitándose el costo de realizar otros exámenes innecesarios así como entregar asesoría genética adecuada y oportuna a la familia.

\section{Referencias}

1. Boue J, Bou A, Lazar P. Retrospective and prospective epidemiological studies of 1500 karyotyped spontaneous human abortions. Teratology 1975; 12: 11-26.

2. Hassold T, Chen N, Funkhouser J, Jooss T, Manuel B, Matsuura J, et al. A cytogenetic study of 1000 spontaneous abortions. Ann Hum Genet 1980; 44: 151-78.

3. Jacobs PA, Browne C, Gregson N, Joyce C, White H. Es- 
Caracterización citogenético-molecular de enfermedades genéticas en Chile - M. A. Alliende et al

timates of the frequency of chromosome abnormalities detectable in unselected newborns using moderate levels of banding. J Med Genet 1992; 29: 103-8.

4. Nazer J, Antolini M, Juárez M, Cifuentes L, Hubner ME, Pardo A. et al. Prevalencia al nacimiento de aberraciones cromosómicas en el Hospital Clínico de la Universidad de Chile: período 1990-2001. Rev Med Chile 2003; 131: 651-8.

5. Bache I, Hjorth M, Bugge M, Holstebroe R, Hilden R, Schmidt L. Systematic re-examination of carriers of balanced reciprocal translocations: a strategy to search for candidate regions for common and complex diseases. Eur J Hum Genet 2006; 14: 410-7.

6. Swerdlow AJ, Schoemaker MJ, Higgins CD, Wright AF, Jacobs PA. Mortality risks in patients with constitutional autosomal chromosome deletions in Britain: a cohort study. Hum Genet 2008; 123: 215-24.

7. Rauch A, Hoyer J, Guth S, Zweier C, Kraus C, Becker C. Diagnostic yield of various genetic approaches in patients with unexplained developmental delay or mental retardation. Am J Med Genet 2006;140: 2063-74.

8. Wincent J, Anderlid BM, Lagerberg M, Nordenskjöld M, Schoumans J. High-resolution molecular karyotyping in patients with developmental delay and/or multiple congenital anomalies in a clinical setting. Clin Genet 2010.

9. Schoumans J, Ruivenkamp C. Laboratory methods for the detection of chromosomal abnormalities Methods Mol Biol 2010; 628: 53-73.

10. Lagos LM, Poggi MH. Tests genéticos: Definición, métodos, validación y utilidad clínica. Rev Med Chile 2010; 138: 1128-32.

11. Castillo S, Fuentes AM, Paulos A, Pardo A. Múltiple FISH y múltiple BAND: técnicas de citogenética molecular en cinco casos. Rev Med Chile 2002; 130: 511-8.

12. Speicher MR, Gwyn Ballard S, Ward DC. Karyotyping human chromosomes by combinatorial multi-fluor FISH. Nature Genet 1996; 12: 368-75.

13. De Vries BB, Pfundt R, Leisink M, Koolen DA, Vissers Le, Janssen IM. Diagnostic genome profiling in mental retardation Am J Hum Genet 2005; 77: 606-16.

14. Shaffer LG, American College of Medical Genetics Professional Practice and Guidelines Committee: American college of medical genetics guideline on the cytogenetic evaluation of the individual with developmental delay or mental retardation. Genet Med 2005; 7: 650-4.

15. Curry CJ, Stevenson RE, Aughton D, Byrne J, Carey JC, Cassidy S, et al. Evaluation of mental retardation: Recommendations of a Consensus Conference: American College of Medical Genetics. Am J Med Genet 1997; 72: 468-77.
16. Battaglia A, Bianchini E, Carey JC. Diagnostic yield of the comprehensive assessment of developmental delay/ mental retardation in an institute of child neuropsychiatry. Am J Med Genet 1999; 82: 60-6.

17. Bugge M, Bruun-Petersen G, Brøndum-Nielsen K, Friedrich $U$, Jensen $G$, Jensen PKA, et al. Disease associated balanced chromosome rearrangements: a resource for large scale genotype-phenotype delineation in man. J Med Genet 2000; 37: 858-65.

18. Alliende MA, Cámpora L, Curotto B, Toro J, Valiente A, Castillo $\mathrm{M}$, et al. Búsqueda de afecciones genéticas como etiología de déficit intelectual en individuos que asisten a escuelas de educación especial. Rev Med Chile 2008; 136: 1542-51.

19. Szot J. Mortalidad infantil por malformaciones congénitas: Chile, 1985-2001. Rev Chil Pediatr 2004; 75: 347-54.

20. Aracena M. Cardiopatías congénitas y síndromes malformativos genéticos. Rev Chil Pediatr 2003; 74: 426-31.

21. Finucane B, Haas-Givler B, Simon EW. Genetics, mental retardation, and the forging of new alliances. Am J Med Genet 2003; 117: 66-72.

22. Estay A, Parra R, Benítez H. Alteraciones cromosómicas en linfocitos de sangre periférica. Rev Chil Pediatr 2007; 78: 363-8.

23. Castillo S, Tobella L, Salazar S, Daher V, Sanz P, López F, et al. Alteraciones cromosómicas en niños referidos para estudio citogenético. Rev Chil Pediatr 1994; 65: 210-4.

24. Be C, Velásquez P, Youlton R. Laboratorio de citogenética: Experiencia de 15 años. Rev Med Clínica Las Condes 1999; 10 (1) Disponible en: http://www.clinicalascondes. cl/

25. Santa María L, Curotto B, Cortés F, Rojas C, Alliende MA. [Molecular diagnosis of Prader-Willi and Angelman syndromes: methylation, cytogenetics and FISH analysis]. Rev Med Chile 2001; 129: 367-74.

26. Kosaki K, Mcginniss MJ, Veraksa AN, Mcginnis WJ, Jones KL. Prader-Willi and Angelman syndromes: diagnosis with a bisulfite-treated methylation-specific PCR method. Am J Med Genet 1997; 73: 308-13.

27. Saluto A, Brussino A, Tassone F, Arduino C, Cagnoli C, Papi $\mathrm{P}$, et al. An enhanced polymerase chain reaction assay to detect pre- and full mutation alleles of the Fragile X Mental Retardation 1 gene. J Mol Diagn 2005; 7: 605-2.

28. Tassone F, Pan R, Amiri K, Taylor AK, Hagerman PJ. A Rapid Polymerase Chain Reaction-Based Screening Method for Identification of All Expanded Alleles of the Fragile X (FMR1) Gene in Newborn and High-Risk Populations. J Mol Diagn 2008; 10: 43-9.

29. Castillo S. Consejo genético. Rev Chil Pediatr 1993; 64: 34-7. 
30. Dinesh RD, Pavithran K, Henry PY, Elizabeth KE, Sindhu P, Vijayakumar T. Correlation of age and birth order of parents with chromosomal anomalies in children Genetika 2003; 39: 834-9.

31. Cruz-Coke R, Moreno RS. Genetic epidemiology of single gene defects in Chile. J Med Genet 1994; 31: 702-6.

32. Rojas CV, María LS, Santos JL, Cortés F, Alliende MA. A frameshift insertion in the cone cyclic nucleotide gated cation channel causes complete achromatopsia in a consanguineous family from a rural isolate. Eur J Hum
Genet 2002; 10: 638-42.

33. Brent RL. Environmental causes of human congenital malformations: The pediatrician's role in dealing with these complex clinical problems caused by a multiplicity of environmental and genetic factors. Pediatrics 2004; 113: 957-68.

34. Cassidy SB, Gaineyj AJ, Butler MG. Occupational hydrocarbon exposure among fathers of Prader-Willi syndrome patients with and without deletions of 15q. Am J Hum Genet 1989; 44: 806-10. 